\title{
Detection of bioactive compounds in persimmon (Diospyros kaki) using UPLC-ESI- Orbitrap-MS/MS and fluorescence analyses
}

\begin{abstract}
Persimmon (Diospyros kakiL.) contains a number of metabolites, especially polyphenols and carotenoids, and persimmon is one of the most healthy fruits linking to a diet with reduced incidences of several diseases. However, little data are available on the persimmon in connection with its metabolites and properties. In recent study the differences between polar and non-polar extracts by1H NMR-based metabolomics was done. The present work presents a complete qualitative analytical characterization by UPLC-ESI-Orbit rap-MS/MS analysis and fluorescence studies in six persimmon cultivars. The metabolic differences were shown as well in the results of bioactivities and antioxidant capacities. The results of binding properties of persimmon polyphenols towards human serum albumin support polyphenolalbumin interactions with human plasma and characteristic to other plants. The presented methods can be widely used for quantitation of multiple compounds in many plants and biological samples, especially in vegetables and fruits
\end{abstract}

Keyword: Diospyros kaki L.; Metabolites; UPLC-ESI-Orbitrap-MS/MS; Polyphenols; Antioxidants; Fluorescence 